$37 \mid 2006$

Langue(s) et religion(s) : une relation complexe dans l'enseignement du français hors de France $x V l^{e}-x X^{e}$ siècle

\title{
Exils et exportation de la langue française : huguenots et congréganistes, 1685-1914
}

\section{Patrick Cabanel}

\section{QpenEdition}

\section{Journals}

Édition électronique

URL : https://journals.openedition.org/dhfles/64

DOI : $10.4000 /$ dhfles. 64

ISSN : 2221-4038

Éditeur

Société Internationale pour l'Histoire du Français Langue Étrangère ou Seconde

Édition imprimée

Date de publication : 1 décembre 2006

Pagination : 11-24

ISSN : 0992-7654

Référence électronique

Patrick Cabanel, « Exils et exportation de la langue française : huguenots et congréganistes,

1685-1914 », Documents pour l'histoire du français langue étrangère ou seconde [En ligne], 37 | 2006, mis en ligne le 01 juillet 2009, consulté le 27 mai 2021. URL : http://journals.openedition.org/dhfles/64 DOI : https://doi.org/10.4000/dhfles.64 


\section{Exils et exportation de la langue française : huguenots et congréganistes, 1685-1914}

Université de Toulouse-Le Mirail L'histoire du rayonnement international de la langue française n'est pas liée au seul
prestige de ses écrivains ou à l'expansion de l'empire colonial : le français a été
également disséminé par des exilés. Les protestants du Refuge huguenot au
lendemain de 1685 , les membres des congrégations religieuses au début du XX
siècle, ont choisi de tourner le dos à une France qui niait leurs droits, mais ont
emporté sa langue et lui ont offert un brillant destin européen ou proche-oriental.
Paradoxale diffusion: le bonheur d'une langue épouse le malheur d'une partie de ses
enseignants et de ses locuteurs.

The history of the international prominence of the French language is not linked to the sole prestige of its writers or to the expansion of the colonial Empire, but more particularly to the exile of part of its population. The protestants belonging to the Huguenot Refuge in 1685, as well as the members of the Catholic congregations at the beginning of the 1900's, chose to turn their back on France, a country that deprived them of their civil rights. At the same time they took their language with them, offering it eventually a glorious destiny throughout Europe and the Near East.

Quels qu'aient été les moyens de la puissance linguistique et culturelle au temps du Grand Siècle ou de l'Empire colonial, ni le XVIII ${ }^{\mathrm{e}}$ ni le $\mathrm{XX}^{\mathrm{e}}$ siècle n'auraient vu à ce point l'Europe ou la Méditerranée parler français, s'il n'y avait eu à l'origine, Refuge huguenot ou exil congréganiste, une dispersion contrainte d'un ensemble de Français, les 
quelque 200 à 250000 protestants après la révocation de l'édit de Nantes (1685), les quelques 30000 religieuses et religieux après les lois hostiles aux congrégations (1901-1904). Le malheur de ces minoritaires ou bannis a puissamment contribué à faire le bonheur de leur langue. On voit combien est paradoxal ce paysage de l'expansion linguistique française. Il n'y a certes rien de commun entre les aristocraties lettrées de l'Europe du XVIII ${ }^{\mathrm{e}}$ siècle et, deux cents ans plus tard, les bourgeoisies du Levant et parfois même des populations catholiques orientales, musulmanes ou arméniennes au niveau de vie très modeste ; rien, sinon leur goût du français et de la distinction que sa maîtrise procure, et leur réponse à l'offre démultipliée que leur ont proposée des Français fraîchement arrivés en exil, contraints de quitter leur pays mais voyageant avec sa langue et devenus de ce fait les meilleurs agents de sa diffusion et de son rayonnement.

Ces hommes et ces femmes, victimes d'une politique conjoncturelle de la France, l'espéraient provisoire et attendaient l'heure du retour, que les congréganistes eurent le bonheur de connaître dès 1914 ou 1918, mais qui ne devait sonner que bien trop tard pour les huguenots, puisque le décret donnant la nationalité française à tout descendant d'exilé pour raison religieuse qui rentrerait en France n'est intervenu qu'en décembre 1790. Chacun n'en a pas moins attendu que le cours de l'histoire se redresse. La traversée du Désert allait prendre fin, le Roi allait rappeler les protestants, ses sujets fidèles entre tous - du moins l'ont-ils cru jusqu'à la paix de Ryswick, en 1697 ; la République allait changer de politique à l'occasion d'élections générales, et rappeler les congréganistes ou les laisser rentrer discrètement, comme cela s'était passé quelques années après les décrets pris en juin 1880 contre les jésuites ${ }^{3}$. Pour l'heure, la langue assurait cette continuité de la France que l'Etat, pour sa part, venait de briser. D'autant que l'on a affaire à des exils constitués pour une forte part de professionnels de la culture et de l'école, enseignants, intellectuels, journalistes, écrivains, "clercs » : la presque totalité des pasteurs et des professeurs d'académies protestantes autour de 1685, des milliers d'enseignants congréganistes de tous niveaux, de l'humble religieuse au grand savant, autour de 1901. Arrivés à l'étranger, ces spécialistes ont cherché à retrouver les

\footnotetext{
${ }^{3}$ Quelque milliers de jésuites et autres religieux avaient alors quitté la France, pour la regagner discrètement moins d'une dizaine d'années plus tard, la République fermant les yeux.
} 
conditions de leur métier, entre pédagogie et vie intellectuelle, et ont recouru aux seuls outils et savoir-faire qu'ils avaient pu emporter, audelà de la religion : la langue et les livres. C'est à l'enseigne du français que beaucoup ont placé leur survie ou leur reconquête d'un statut.

Leur chance a tenu dans le prestige de cette langue et de la France elle-même, en dépit de leur propre statut de vaincus et de réfugiés. C'est là un élément qui distingue les exils de 1685 et de 1901 de ceux d'autres minorités qui ne pouvaient espérer emporter dans leurs bagages une langue prestigieuse ou à l'utilité internationalement reconnue. Ni les hussites, ni les vaudois, ni les Arméniens, ni les Russes blancs, ni les républicains espagnols, ni les Palestiniens, ni même les antinazis ou les Juifs allemands n'ont eu la chance des exilés français qui du fond de leur malheur n'en restaient pas moins pourvus de la première langue d'Europe, et pouvaient monnayer ce privilège : dans le Berlin ou la Rotterdam de 1700 comme dans les Beyrouth, Damas ou Buenos Aires de 1904, l'exilé continuait à parler la langue enviable des maîtres du monde ou d'une partie du monde. Et leur exil même a renforcé le statut international de cette langue, par une sorte d' «immoralité » radicale de l'histoire qui a voulu que les perdants œuvrent gratuitement, en quelque sorte, à répandre dans des pays entiers non pas la religion qui leur valait leur malheur (la Prusse ou les PaysBas de 1700 ne sont pas devenus plus protestants, la Belgique ou l'Italie de 1904 ne sont pas devenues plus catholiques), mais l'empire informel d'une langue et par là, au moins au $\mathrm{XX}^{\mathrm{e}}$ siècle, d'une nation. A moins que l'on ne se réfère à une "moralité » supérieure de l'histoire, $a$ posteriori, en relevant que «bourreaux » et « victimes » ont également servi la France, les uns à l'intérieur, les autres sur les routes de l'exil, et que la mesure des "grands siècles" de la langue française n'est complète que si l'on compte un Bayle aux côtés d'un Bossuet, les Frères des Ecoles chrétiennes émigrés au Liban aux côtés des hussards noirs de métropole ${ }^{4}$. Je propose de risquer cette mesure à propos du Refuge huguenot, puis de l'exil congréganiste ${ }^{5}$.

\footnotetext{
${ }^{4}$ Ce texte reprend en partie un article plus ancien, qui traitait également de l'Alliance israélite universelle, "Langue et diasporas. Trois exemples d'expansion paradoxale du français », Diasporas. Histoire et sociétés, n², 2003, p. 102-119. Voir aussi l'ouvrage que j'ai dirigé, Une France en Méditerranée. Ecoles, langue et culture françaises XIX $X^{e}-X X^{e}$ siècles, Grâne : Créaphis, 2006.

${ }^{5}$ Un panorama complet amènerait à s'intéresser également à l'émigration française sous la Révolution, avec son fort contingent ecclésiastique.
} 


\section{Le Refuge huguenot}

Dans le Refuge huguenot, de nombreux pasteurs, auxquels les paroisses de l'Europe protestante, déjà bien pourvues, ne pouvaient assurer un ministère, se sont faits précepteurs, traducteurs, journalistes, directeurs de revues, écrivains. On a établi que $78 \%$ des pasteurs en poste en France en 1685 (680 sur 870), ont gagné le Refuge, dont 405 pour les seules Provinces-Unies. A leurs côtés se trouvent des magistrats, militaires, intellectuels divers, confrontés aux mêmes contraintes, riches des mêmes spécialités, qui par goût et par nécessité contribuent à faire $\mathrm{du}$ français, avec le latin, la lingua franca du monde cosmopolite des intellectuels européens, justement désigné par le réfugié de Rotterdam, Pierre Bayle, fils et frère de pasteur, comme « République des lettres ». $\mathrm{Au}$ sein des générations suivantes, les anciens élèves du Collège français de Berlin, lorsqu'ils ne deviennent pas pasteurs, s'efforcent d'obtenir un poste de précepteur dans les familles aristocratiques ou bourgeoises de Stettin, Leipzig, Gotha, Hanau, Riga, Copenhague, Moscou, SaintPétersbourg. On leur demande de pouvoir enseigner à peu près tout, $\mathrm{y}$ compris les mathématiques, mais le français est toujours exigé en première place. "Le principal de tout est la langue française que l'informateur doit savoir et parler comme sa langue maternelle », exige un conseiller de chancellerie de Riga en 1763. Beaucoup de demandes et d'offres passent par l'intermédiaire de Jean-Louis Samuel Formey (1711-1797), le secrétaire perpétuel de l'Académie royale de Berlin, luimême d'origine huguenote, dont les archives conservent des lettres de près de 700 correspondants, venus solliciter une place, pour les uns, la proposer, pour d'autres ${ }^{6}$.

Toutefois, l'œuvre d'assimilation linguistique, sauf peut-être dans les élites, a été très rapide : les huguenots sont rapidement passés à l'allemand, au néerlandais, à l'anglais. Parce que leur intégration était voulue à la fois par eux-mêmes et par les sociétés d'accueil, la langue française ne pouvait guère survivre que comme un reste, condamné à s'user très vite d'une génération à l'autre ou à se figer comme un titre

${ }^{6}$ H. Duranton, «"Un métier de chien”. Précepteurs, demoiselles de compagnie et bohême littéraire dans le Refuge allemand », Dix-Huitième Siècle, 1985, n 17, p. 297-315. 
ancien de noblesse ou d'identité. Alors que rapidement des mots allemands sont entrés dans le français des huguenots, ce dernier n'a subsisté, à Berlin, que sous la forme d'apports lexicaux isolés, dont on remarque que plusieurs ont trait à l'alimentation, ce lieu par excellence des emprunts et des apports. Bulette (boulette), Butelje (bouteille), Stampe (estaminet), et plus encore ces délicieux Bulljong (bouillon) et Ragufeng (ragoût fin) ${ }^{\top}$ rappellent combien la différence est d'abord d'ordre culinaire, et s'exprime dans les mots de la cuisine immigrée. La survie du français a été plus durable, parfois jusqu'à la fin du XIX ${ }^{\mathrm{e}}$ siècle ou même jusqu'à nos jours, pour les usages liturgiques et les lectures religieuses, car c'est le seul domaine, sans doute, où les communautés réfugiées ont continué à vivre de manière séparée, dans les paroisses wallonnes ou huguenotes d'Allemagne et de Hollande.

$\mathrm{La}$ véritable extension de la langue française se trouve dans l'univers des idées. Les pasteurs réfugiés, rompus à l'usage des langues mortes et vivantes, par métier autant que par nécessité désormais, se sont installés aux interstices du latin et du français, d'une part, et des langues européennes, anglais, allemand, néerlandais, italien, de l'autre. «Plénipotentiaires» de chacun des deux univers, pour reprendre la formule qu'emploie Pierre Coste, réfugié d'Uzès, dans sa traduction de John Locke ${ }^{8}$, ils contribuent à offrir le français à l'Europe, en particulier en Hollande et à Berlin, mais aussi l'Europe au français. Parmi les traducteurs connus du XVIII ${ }^{\mathrm{e}}$ siècle, mentionnés par Alexandre Cioranescu ${ }^{9}$, sept sont nés à l'étranger et morts en France, vingt-cinq nés et morts à l'étranger, vingt-neuf nés en France et morts à l'étranger, ces derniers, et une grande partie des seconds, étant des huguenots ${ }^{10}$.

7 J'emprunte cette liste à F. Hartweg, «Les huguenots en Allemagne : une minorité entre deux cultures », M. Madgelaine, R. von Thadden, Le Refuge huguenot, Paris : A. Colin, 1985, p. 198.

${ }^{8}$ P. Coste, Avertissement de la traduction de l'Essai philosophique concernant l'entendement humain, Amsterdam, 1700, cité par P. Hazard, La crise de la conscience européenne, 1680-1715, Le Livre de Poche, "Références », 1994 [1961], p. 73-74.

${ }^{9}$ A. Cioranescu, Bibliographie de la littérature française du dix-huitième siècle, Paris : CNRS, 1969, 3 vol.

10 J'emprunte cette statistique à F. Nies, «La mer à boire ? Conclusion et perspectives », Revue d'Histoire littéraire de la France, mai-juin 1997, "Les traductions dans le patrimoine français », p. 448-460 [note 37, p. 458]. Parmi les étrangers issus du Refuge, S.H. Catel, de la troisième génération, traduit en allemand le Dictionnaire de l'Académie française (Berlin, 4 vol., 1800-1801). 
Des travaux récents ont mis en valeur leur rôle ${ }^{11}$. Ils fondent divers périodiques qui s'efforcent de présenter la pensée vivante dans diverses aires culturelles : Bibliothèque anglaise (Amsterdam, 1717-1728) puis Bibliothèque britannique (La Haye, 1733-1747) et Journal britannique (La Haye, 1750-1757) ; Bibliothèque italique (Genève, 1728-1734); Bibliothèque germanique (Amsterdam, 1720-1740) et ses continuations jusqu'en 1759. Ils traduisent en français Locke (Pierre Coste), Pufendorf et Grotius (Jean Barbeyrac), Spinoza (Gabriel de Saint-Glain), Pestalozzi (Louis Esaïe Pajon de Montcets). Abel Boyer, né à Castres, étudiant en théologie devenu traducteur et précepteur, propose aux lecteurs anglais un Compleat French master for ladies and gentlemen (1694) et The Royal Dictionary (French-English, English-French) (1699), appelé à de nombreuses rééditions. On mesure, face à ces titres de périodiques et d'ouvrages et à l'ampleur encyclopédique des curiosités qu'ils prétendent embrasser, l'importance de ce qui s'est passé dans l'Europe des années $1685-1750$, de ce qui a transité d'une langue à l'autre. Que l'on songe à l'essor de la réflexion politique française au $\mathrm{XVIII}^{\mathrm{e}}$ siècle, de Montesquieu à Rousseau, si Locke n'avait été aussi rapidement traduit par un exilé huguenot.

On peut s'interroger, toutefois, sur l'identité des véritables bénéficiaires en matière de diasporas et d'influences linguistiques et culturelles. Sont-ce vraiment la langue et le pays de départ, l'Espagne «privée» de son judaïsme mais dont la langue parcourt les rives méditerranéennes sous la forme du judéo-espagnol, la France amputée d'une part notable de son protestantisme et par là peut-être de ses élites, mais dont la langue irrigue les cours et les villes de l'Europe du nord? Des historiens juifs et protestants, au XIX ${ }^{\mathrm{e}}$ siècle, ont affirmé leur croyance dans une sorte de justice immanente qui serait venue frapper les nations coupables d'avoir sacrifié leurs minorités sur l'autel du rêve unitaire catholique : l'Espagne enfoncée dans l'archaïsme et dans la violence politique endémique, la France défaite en 1870 (ou égarée dans un autre rêve unitaire sanglant, en 1793, celui de la Terreur anticatholique), vérifieraient cette règle d'airain, tandis que les nations accueillantes aux diasporas, tels les Pays-Bas, auraient trouvé dans leur

\footnotetext{
${ }^{11}$ La Vie intellectuelle aux Refuges protestants, actes de la Table ronde de Münster du 25 juillet 1995 réunis par J. Häseler et A. McKenna, Paris : Champion, 1999, et La Vie intellectuelle aux Refuges protestants II Huguenots traducteurs, actes de la Table ronde de Dublin, juillet 1999 édités par J. Häseler et A. McKenna, Paris : Champion, 2002.
} 
geste la source d'un épanouissement sans précédent. Un texte malicieux de Paul Hazard donne à réfléchir sur ce point ; l'historien montre l'éclat sans précédent de la vie intellectuelle dans l'Angleterre des années 1700 , et sa conquête de l'Europe dès lors qu'a pu être levé le seul obstacle qui faisait barrage à son rayonnement, la méconnaissance générale de la langue anglaise. Ce sont les traducteurs et autres intermédiaires huguenots réfugiés en Angleterre qui ont mis ses idées à disposition de l'Europe ${ }^{12}$. Ce faisant, ils n'œuvraient pas plus pour l'Angleterre que contre la France dont l'intolérance les avait forcés à s'enfuir. L'idée moderne de nation n'avait pas encore surgi, et l'on sait que de très nombreux officiers huguenots ont servi des armées étrangères sans trouble de conscience. L'identité confessionnelle était probablement plus forte que l'identité « nationale » (ce mot même est un anachronisme), et la langue française n'avait pas encore été rattrapée par le nationalisme : elle était alors, outre la langue maternelle des réfugiés et celle dans laquelle ils se préoccupaient de leur salut ${ }^{13}$, une langue de travail et de communication en Europe. On n'imagine pas un Louis XIV se réjouissant d'apprendre que, grâce aux Jurieu et Bayle, les grands adversaires de sa politique et de sa philosophie, on parle français dans toutes les cours et revues savantes du continent; pas plus que les reyes catolicos n'ont dû se féliciter de ce que les Juifs chassés d'Espagne en 1492 aient implanté leur langue à Salonique: il a fallu attendre le premier tiers du $\mathrm{XX}^{\mathrm{e}}$ siècle pour qu'une Espagne malade de sa défaite à Cuba (1898) et de l'archaïsme de ses structures socio-économiques et culturelles, se prenne à songer, dans des milieux du reste très circonscrits, à la carte que pourrait représenter la diaspora sépharade. Le sarcasme d'un Voltaire à l'encontre du "style réfugié », ce français archaïsant et hébraïsant écrit (plus que parlé) dans le Berlin de son époque, suffit à marquer l'absence de conscience de ce que nous appellerions aujourd'hui une francophonie.

\section{L'exil congréganiste}

\footnotetext{
${ }^{12}$ P. Hazard, op. cit., p. 70-71.

${ }^{13}$ Pour les huguenots issus des milieux paysans du Midi occitanophone, elle n'était que la langue de Dieu, si l'on peut dire.
} 
Les choses se présentent tout autrement avec la diaspora congréganiste au début du $\mathrm{XX}^{\mathrm{e}}$ siècle. Ses effectifs sont beaucoup plus réduits; à la vérité, on ne les connaît que de manière approximative : La Croix a avancé, le 5 août 1914, le chiffre de 60000 exilés. Diverses extrapolations, pays par pays ou congrégation par congrégation, permettent de penser qu'au moins 30000 religieux des deux sexes ont gagné l'étranger. La vague migratoire s'amorce dès l'été 1901, lorsque les jésuites et d'autres instituts renoncent à demander l'autorisation requise par la nouvelle loi sur les associations (elle est effectivement refusée, l'année suivante, à la quasi totalité des congrégations qui ont déposé des demandes en règle); elle enfle au lendemain de la loi de juillet 1904 qui interdit tout enseignement aux congréganistes et frappe, par exemple, les Frères des Ecoles chrétiennes, dûment autorisés depuis le $\mathrm{XIX}^{\mathrm{e}}$ siècle. Elle est donc composée, pour l'essentiel, d'enseignants : ce sont des milliers d'instituteurs et de professeurs, hommes et femmes, qui ont quitté la France pour sauver leur vocation religieuse et ce qu'elle impliquait de vie communautaire sous l'habit et la règle. Les congrégations déjà implantées à l'étranger ont renforcé leurs établissements avec les nouveaux arrivants, et mis à profit réputation et réseaux pour se voir confier la direction de nouvelles écoles. Certaines, fortes de leur tradition internationale ou missionnaire, ont choisi de diversifier leurs implantations et d'explorer de nouvelles destinations, en Europe, au Levant, dans les deux Amériques. Vivre en exil, pour un couvent enseignant ou une poignée de frères ou religieuses, consiste à ouvrir une école et à s'attacher une clientèle, quitte à commencer par l'accueil gratuit des pauvres et en faisant porter ses espérances sur la bourgeoisie susceptible de verser des sommes convenables et d'accroître le prestige de l'établissement.

Si l'enseignement dans les écoles gratuites, au moins au départ, fait la plus large place à la langue du pays d'accueil, comme l'espagnol dont 360 Frères des Ecoles chrétiennes destinés à l'Amérique latine ont appris les rudiments dans un noviciat spécial, à Clermont-Ferrand (fermé en 1909), les écoles payantes et autres pensionnats proposent à leurs élèves et aux familles bourgeoises la suprême distinction du français. Ainsi s'explique que les élites d'Amérique latine, du Liban, de la Syrie, de l'Egypte pour partie, voire de la vieille Europe, déjà familières des collèges des hermanos lasallistas (Frères des Ecoles chrétiennes) ou padres franceses (Picpuciens, Pères du Sacré-Cœur de 
Bétharram, etc.), pour les premières ${ }^{14}$, aient achevé de devenir francophones dans leur scolarité puis dans leur vie sociale et parfois familiale. En 1913, le Levant réunit pas moins de 830 Frères des Ecoles chrétiennes, accueillant au total plus de 14000 élèves; l'Amérique latine 978 frères et 23664 élèves, le Canada 748 frères et 22252 élèves, la Belgique 1281 frères et 30067 élèves : les religieux sont autant d'agents de l'expansion du français. A leurs côtés, parfois en concurrence avec eux, on trouve des dizaines d'autres congrégations d'hommes et de femmes. Jules Cambon, ambassadeur de France aux Etats-Unis, a fait connaître au ministère, en 1901, que si les religieuses américaines scolarisent 516100 élèves, les Françaises installées sur place en accueillent pas moins de $235202^{15}$.

Outre la différence des conditions sociales et culturelles - une élite d'intellectuels à la fin du XVII ${ }^{\mathrm{e}}$ siècle, une masse d'enseignants du primaire et du secondaire au début du $\mathrm{XX}^{\mathrm{e}}$-, un trait politique majeur distingue les diasporas huguenote et congréganiste: la seconde se développe dans une Europe et une Méditerranée marquées par le phénomène national et le choc de rivalités impériales qui sont aussi des rivalités scolaires et linguistiques. Or la France, moins bien lotie en termes de puissance démographique (et donc migratoire), économique et commerciale, attend d'autant plus de la puissance de sa langue, fût-elle servie par des adversaires exilés. Des équations sont censées s'imposer encore dans les années 1900, au moins dans la mosaïque nationale et confessionnelle du Levant: être catholique, c'est être français ou protégé ou client de la France (et vice-versa); et parler français, plus encore. On comprend l'intérêt, aux yeux de Paris, de ces congréganistes qui scolarisent en français quelques centaines de milliers d'élèves dans le monde: ils arraisonnent à la France de nouvelles provinces. La République laïque, à cet égard, semble avoir agi avec plus d'intelligence ou de cynisme que Louis XIV - ou du moins savait-elle combien la puissance relative du pays avait reculé : elle a utilisé les congréganistes,

\footnotetext{
${ }^{14}$ Sur cette influence et son épuisement dans la première moitié du $\mathrm{XX}^{\mathrm{e}}$ siècle, G. Matthieu, Une ambition sud-américaine. Politique culturelle de la France (19141940), Paris: L'Harmattan, 1991, et D. Rolland, La crise du modèle français. Marianne et l'Amérique latine. Culture, politique et identité, Rennes: Presses Universitaires de Rennes, 2000.

${ }^{15}$ Note du 12 juin 1901, résumée dans une Note pour le directeur des Affaires politiques, 17 novembre 1904, Paris. Archives du Ministère des Affaires étrangères, Saint-Siège Nouvelle Série 23, fo 249.
} 
et considéré que leurs réseaux et leurs statistiques étaient, objectivement, les siens. Aussi les religieux exilés ont-ils agi sous la protection des ambassadeurs et consuls de France, et bénéficié d'une manne financière qui leur était très officiellement destinée, année après année, à la suite du vote du budget du ministère des Affaires étrangères. Diplomates et ministres insistent alors à l'envi sur l'extraordinaire instrument de rayonnement que les religieux missionnaires et exilés mettent presque gratuitement à la disposition de la France et qu'ils défendent contre les appétits italiens, allemands, américains ou britanniques. Alors qu'une minorité ultra-laïque réclame la cessation des subventions aux écoles congréganistes, les ministres, à commencer par Théophile Delcassé dans le gouvernement d'Emile Combes, montent au créneau pour sauver ces subventions au nom de l'intérêt supérieur linguistique - de la nation. Ce sont environ 750000 francs qui sont destinés chaque année aux écoles congréganistes. En 1909, la direction politique du ministère des Affaires étrangères se dote d'un Service des écoles et des œuvres, ébauche de la future Direction générale des affaires culturelles, qui achève d'officialiser le soutien financier et politique, par le plus laïque des régimes, à l'enseignement congréganiste qu'il a chassé de son sol et aux exilés qui ont refusé de se soumettre à ses lois ${ }^{16}$ !

Les religieux, pour leur part, font passer au second plan le prosélytisme (pratiquement voué à l'échec, du reste, dans le monde musulman), mettent en sourdine leur hostilité à la République et à la laïcité, et se présentent devant les autorités françaises comme les meilleurs missionnaires de la langue et de la nation. Ce n'est pas seulement de leur part un prétexte habile ou un glissement sémantique, mais un sentiment largement intériorisé, voire avivé par l'exil : l'identité nationale est maintenant assez forte pour s'imposer à des hommes et des femmes pourtant volontairement partis en exil. Leurs professions de foi nationale sont relayées en France par un certain nombre de grandes voix catholiques, dont un Maurice Barrès qui poursuit le combat jusqu'à son dernier souffle, en $1923^{17}$. Entre cent autres, citons ce passage du catholique libéral et dreyfusard Anatole Leroy-Beaulieu, en 1903 ; après

\footnotetext{
${ }^{16}$ Sur ce thème, je prends la liberté de renvoyer à mon ouvrage, Entre religions et laïcité : la voie française, XIX $-X X^{e}$ siècles, Toulouse : Privat, 2007.

${ }^{17}$ En soutenant un projet de loi visant à favoriser le recrutement en France des congrégations missionnaires, au seul titre de l'expansion de l'influence et de la langue françaises.
} 
avoir salué «des conquérants ambitieux de conquérir de nouvelles provinces à la France ou à la langue française, en même temps qu'à l'Evangile », il les montre à l'œuvre dans la «Babel orientale des rivages asiatiques de la Méditerranée $»$ :

Nos religieux y sont partout des missionnaires de notre langue; si, des bouches du Nil aux Dardanelles et au Bosphore, le français l'emporte sur tous ses concurrents, anciens ou nouveaux, sur l'anglais, sur l'allemand, sur l'italien, sur le russe, l'honneur en revient, avant tout, à ces Pères, à ces Frères, à ces Sœurs, odieusement vilipendés par une presse haineuse ${ }^{18}$.

La République laïque a entendu ce type d'arguments, persuadée que l'ancienne «clientèle catholique» de la France au Levant, pour reprendre une formule de Gambetta, allait devenir une «clientèle francophone »- ce dernier mot existe désormais, forgé, on le sait, par Onésime Reclus en 1880. On peut dire qu'il y a à l'étranger une sorte d'union sacrée autour de la puissance linguistique de la France, une collusion objective et même officialisée entre un Etat laïque et ses opposants cléricaux. L'aile la plus résolue du camp anticlérical a toutefois fondé, en 1902, la Mission laïque et l'Ecole normale JulesFerry (Paris), destinées à former et envoyer dans les colonies et en territoires d'influence française des instituteurs nourris des valeurs de la France laïque moderne. Les premiers établissements sont implantés dans l'Orient méditerranéen, si fortement marqué par l'action des congréganistes : dès lors, à Salonique comme à Beyrouth, expatriés de la Mission laïque et exilés des congrégations enseignantes se font face, chacun parvenant à conquérir et fidéliser une clientèle avide de culture française. Les enseignants juifs de l'Alliance israélite universelle, j'y reviens dans un instant, sont également actifs, et encore au bénéfice du français.

\section{Conclusion}

Le rayonnement international du français, au cours des siècles, s'explique par bien des raisons sur lesquelles il est impossible d'insister

\footnotetext{
${ }^{18}$ A. Leroy-Beaulieu, « Les congrégations religieuses. Le protectorat catholique et l'influence française au dehors », Revue des Deux Mondes, $1^{\text {er }}$ mars 1903, p. 81.
} 
ici : puissance démographique, politique et militaire de la France de Louis XIV puis de la Révolution et de Napoléon ; gloire des écrivains du Grand Siècle puis des Lumières ; nécessité permanente, entre Etats et voyageurs, de recourir à une langue commune utilitaire, qui fut le latin, est aujourd'hui l'anglais, mais a longtemps été le français ; éléments de snobisme, aussi. Ces traits renvoient à un long $\mathrm{XVIII}^{\mathrm{e}}$ siècle, cher à Marc Fumaroli ${ }^{19}$. A la fin du XIX ${ }^{\mathrm{e}}$ siècle, le rayonnement du français s'est déplacé, dans ses zones de force comme dans ses vecteurs. L'Europe germanique lui échappe presque complètement désormais, mais l'Europe balkanique et le monde méditerranéen, du Maghreb au Levant, voire l'Amérique latine, ont pris le relais. Terre d'asile des militants des causes « nationalitaires », la France a accueilli et formé des Grecs, Italiens, Polonais, Roumains, Tchèques ; on a pu dire de la jeune Roumanie qu'elle représentait probablement «la plus belle réussite d'influence par la culture jamais enregistrée dans l'histoire moderne ${ }^{20} »$. La colonisation, bien sûr, entraîne la mise en place de réseaux scolaires, éditoriaux, culturels, en langue française, de l'Algérie aux protectorats et territoires de mandat. En 1883, l'Alliance française a entrepris de bâtir son propre réseau, tandis que l'Etat fonde des centres culturels et des Ecoles françaises à l'étranger, en attendant la Mission laïque. Les Juifs français, enfin, ont fondé en 1860 et n'ont cessé de développer l'Alliance israélite universelle, qui s'est bientôt trouvée à la tête d'un imposant réseau scolaire, du Maroc aux Balkans, et a fait du français, sauf exception, la langue de ses enseignants (formés à Paris et à Auteuil) et de ses élèves.

Les divisions entre les trois réseaux, catholique, laïque, juif, ont été volontiers soulignées. La Mission laïque et les congrégations se frappent mutuellement d'anathème, au nom de la conquête par la France des clientèles coloniales et orientales. Seule la France laïque peut accueillir toutes les religions sans en effaroucher aucune, disent les uns ; seule l'école catholique peut être comprise par des Orientaux aux yeux desquels religion et nation sont étroitement associées, rétorquent les

\footnotetext{
${ }^{19}$ M. Fumaroli, Quand l'Europe parlait français, Paris : Editions de Fallois, 2001

${ }^{20}$ Neagu Djuvara, cité par A. Niculescu, Aux racines de la démocratie en Roumanie: Pruncul Român (L'Enfant Roumain), premier journal libre roumain, chronique de la révolution valaque de 1848, Thèse, Univ. Montpellier-III, 2002, p. 94. Voir P. Eliade, De l'influence française sur l'esprit public en Roumanie, Paris : Leroux, 1898.
} 
autres. A la tribune de la Chambre des députés ou du Sénat, des catholiques dénoncent le soutien de fait de la République à l'Alliance israélite $^{21}$, ou l'appartenance au judaïsme de tel dirigeant ou de telle clientèle locale de la Mission laïque. Au Sénat, le sénateur catholique Jenouvrier s'exclame, en décembre 1907: «Ainsi, c'est cela la France $\mathrm{du} \mathrm{XX}^{\mathrm{e}}$ siècle $[\ldots]$; les établissements qui doivent avoir la préférence de notre Gouvernement ou de notre budget sont ceux qui relèvent de l'Alliance israélite universelle qui n'est pas française et qui n'est même pas neutre au point de vue confessionnel». Au cours de la même discussion, le ministre Stephen Pichon ayant vanté la réussite de la Mission laïque à Salonique, l'amiral de Cuverville lui demande la nationalité des élèves et son collègue Gaudin de Villaine s'exclame : «Ce sont tous des Juifs ! ${ }^{22}$. Il est vrai que l'historien libre penseur Alphonse Aulard, président de la Mission laïque, venait de saluer, au retour d'un voyage en Turquie et en Egypte, ces écoles de l'Alliance

où on enseigne la langue et la culture françaises dans un esprit vraiment moderne, $[\ldots]$ qui ne nous coûtent pas un sou, et dont l'action a été si efficace pour la substitution de la langue française à la langue italienne ${ }^{23}$.

D'autres observateurs, somme toute proches de la ligne du ministère des Affaires étrangères, qui soutenait à la fois les congrégations et la Mission laïque, ont préféré mettre l'accent sur la complémentarité des réseaux républicains et des diasporas juive ou congréganiste. Le champion d'un œcuménisme linguistique et patriotique est sans doute l'universitaire Henri Hauser, d'origine juive, qui n'hésite pas à prôner le soutien à l'islam en Afrique du Nord, aux congrégations partout où elles ne froissent pas les susceptibilités des populations, à l'Alliance israélite face aux 60000 Juifs de Salonique, aux écoles laïques mises en place par Gallieni à Madagascar, enfin aux missions protestantes à Tahiti et

\footnotetext{
${ }^{21}$ Soutien de fait, non de droit ; en 1868 et 1879, à la demande de l'Alliance, le ministère des Affaires étrangères a recommandé aux consuls de protéger les écoles. Des subventions régulières ne sont versées qu'au lendemain de la Première Guerre mondiale; A. Rodrigue, De l'instruction à l'émancipation. Les enseignants de l'Alliance israélite universelle et les Juifs d'Orient 1860-1939, Paris : CalmannLévy, 1989, p. 18-19, et French Jews, Turkish Jews. The Alliance Israélite Universelle and the Politics of Jewish Schooling in Turkey, 1860-1925, Bloomington \& Indianapolis : Indiana University Press, 1990.

${ }^{22}$ Journal Officiel, Sénat, 25 décembre 1907, p. 1275-1285.

${ }^{23}$ A. Aulard, «Les Frères en Orient », La Dépêche, 24 juillet 1907.
} 
aux Nouvelles-Hébrides ${ }^{24}$ ! Dans un article de la Revue des Deux Mondes, en 1909, Anatole Leroy-Beaulieu salue tour à tour les services rendus à la langue française par les congrégations, l'Alliance israélite et la Mission laïque, et conclut :

Notre langue est pareille à la Semeuse au large geste de nos monnaies nouvelles. Voici bientôt deux siècles qu'elle répand, à pleines mains, sur l'Europe et sur le monde des graines d'idées que les brises de la mer et le vent des montagnes emportent au loin ${ }^{25}$.

Etrange et belle image, à la réflexion, que cette Semeuse chère aux monnaies et aux timbres 1900, ou encore au dictionnaire Larousse : on reconnaît en elle une version de la Marianne républicaine et laïque, mais aussi une figure possible de la diaspora, dissémination et ensemencement des hommes, des idées, des mots. Le français, quelle qu'ait été la force de sa liaison à un territoire, à des frontières « naturelles », à une nation, a été cette langue de diaspora : c'est l'un des privilèges de son histoire tourmentée.

${ }^{24}$ H. Hauser, «Les missions religieuses et l'intérêt national», Annales Coloniales, 15 mai 1904, p. 222-230.

${ }_{25}$ A. Leroy-Beaulieu, "La langue française et les révolutions de l'Orient », Revue des Deux Mondes, 15 avril 1909, p. 871. 